\title{
Spatio-temporal Detachment of Single Cells Using Microarrayed Transparent
}

\section{Electrodes}

Junji Fukuda*, Yoshiaki Kameoka, Hiroaki Suzuki

Graduate School of Pure and Applied Sciences, University of Tsukuba, 1-1-1 Tennodai, Tsukuba, Ibaraki 305-8573, Japan

*Corresponding author: Tel.: +81-29-853-4995; Fax: +81-29-853-4490; E-mail: fukuda@ims.tsukuba.ac.jp

\section{Abstract}

This paper describes a dynamic cell manipulation approach by which cells can be selectively detached from transparent indium tin oxide (ITO) electrodes via electrochemical desorption of a self-assembled monolayer (SAM) of alkanethiol. Changes in the surface properties of ITO following modification and electrical desorption of the SAM were characterized. By using these reactions on ITO, cells were readily attached and then detached from ITO electrodes in a very rapid manner, with greater than $90 \%$ of the cells being detached within 5 min. Furthermore, we fabricated 
micropatterns of ITO electrodes using photolithography. Cells on the micropatterned ITO electrodes could be detached with single-cell resolution. This approach could potentially lead to on-demand harvesting or elimination of one population from others under microscopy, for a wide range of purposes.

\section{Keywords}

Indium tin oxide; electrochemistry; gold-thiolate bond; self-assembled monolayer; cell detachment; tissue engineering 


\section{Introduction}

Development of strategies to design the interface between substrates and cells remains an important challenge in materials science [1]. "Smart" biomaterials that exhibit dynamic changes in interfacial properties, including adherence of cells, are important in a variety of fields, ranging from fundamental cell biology to tissue engineering applications [2-4]. External stimuli such as electrical, thermal, optical, and magnetic fields have typically been used to trigger cell detachment $[5,6]$. For example, by using a culture surface modified with a thermo-responsive polymer, cells can be detached by reducing the temperature from $37^{\circ} \mathrm{C}$ to $20^{\circ} \mathrm{C}$. With this approach, corneal and epithelial cell sheets have been employed for the clinical treatment of corneal disorders and esophageal ulceration following endoscopic surgery, respectively [7, 8]. A magnetic field has also been used to attract magnetically labeled cells on a non-adherent surface and subsequently harvest them as a cell sheet by removal of the magnetic force [9]. These dynamic cell detachment technologies that operate via external stimuli are often characterized by spatial resolution, temporal resolution, and reversibility. Thermal and magnetic approaches typically possess high temporal resolution and reversibility. However, because it is difficult to produce a large difference in thermal and magnetic fields in a limited space, these approaches may not be applicable to selective 
detachment of cells with single-cell resolution. The electrical and optical approaches may possess high spatial and temporal resolutions because localized photo-exposure and fabrication of microelectrode arrays can be achieved with submicron resolution. Electrical approaches for the detachment of cells involve the use of electrically responsive molecules or reactions on the surface of an electrode. For example, two electroactive groups, quinone ester and $O$-silyl hydroquinone, were used to release cell adhesive ligands in response to application of either reductive or oxidative potential, resulting in the selective detachment of adherent cells [10]. Polyelectrolyte layers were also used to electrically detach cells from an electrode [11, 12]. In this approach, electrolysis of water induces local $\mathrm{pH}$ change and subsequent dissolution of the polyelectrolyte layer, resulting in the detachment of cell sheets. Additionally, hydrogel layers were used to detach cells by electrical disruption of the coupling layers [13]. These approaches can achieve selective detachment of cells from a pattern with a resolution of a few hundred micrometers, but higher spatial resolutions for more precise uses such as the detachment of a single cell have not yet been reported.

Our group has also reported on electrochemical approaches by which cells attached to a gold electrode via alkanethiol self-assemble monolayers (SAMs) can be detached via reductive desorption of the SAM by applying a negative electrical potential [14]. Using 
this approach, cells were noninvasively detached within 5 min. Because this approach involves no complex chemistry and enables very rapid cell detachment, it will be useful for various applications $[14,15]$. We considered applying this approach for the spatially selective detachment of cells by fabricating microarrays of gold electrodes and applying a potential to particular microelectrodes. This strategy, however, has a serious practical limitation. Specifically, because there is a large difference in light permeability between glass and gold-coated regions, halation makes it difficult to observe cells on the gold electrodes (Supplemental Fig. 1). Therefore, in the present study, we examined the applicability of transparent ITO electrodes for the detachment of cells along with reductive desorption of the SAM. Furthermore, we investigated whether spatially selective detachment of cells could be conducted while leaving the neighboring cells attached through the use of micropatterned ITO electrodes.

\section{Materials and methods}

\subsection{Materials and reagents}

The reagents used for cell culture and tests were purchased from the following commercial sources: Dulbecco’s modified Eagle medium (DMEM) and fetal bovine serum (FBS) from Invitrogen, USA; endothelial basal medium-2 (EBM-2, CC-3156) 
and SingleQuots growth supplements (CC-3162) from Cambrex Bio Science, USA; fluorescent diacetate (FDA) and ethidium bromide (EB) from Wako Pure Chemicals Industries, Japan; Rhodamine phalloidin from Cytoskeleton, Inc., USA; and 4',6-diamidino-2-phenylindole (DAPI) from Sigma, USA.

Materials used to fabricate the culture substrates were obtained from the following commercial sources: ITO substrate (thickness, $150 \mathrm{~nm}$; resistance, $15 \Omega / \mathrm{cm}$ ) from Sanyo Vacuum Industries, Japan; 10-carboxy-1-decanethiol from Dojindo Laboratories, Japan; GRGDS peptide from the Peptide Institute, Japan; precursor solution of polyimide (Semicofine SP-341) from Toray, Japan; positive photoresist (Shipley 1818) and MF-319 solution from Rohm and Haas, USA; and liquid prepolymers of poly(dimethylsiloxane) (PDMS, KE-1300T) from ShinEtsu Chemical, Japan. All other chemicals were purchased from Sigma, USA, unless otherwise noted.

\subsection{Adsorption and desorption of SAM on ITO}

A circular active area $\left(0.4 \mathrm{~cm}^{2}\right)$ was delineated on an ITO electrode with a photocrosslinkable polyimide insulating layer using a corresponding photomask. The electrode was then cleaned with piranha solution $\left(\mathrm{H}_{2} \mathrm{SO}_{4}: \mathrm{H}_{2} \mathrm{O}_{2}, 3: 1\right)$ and $1.0 \%$ sodium dodecyl sulfate. The electrode surface was covered with a SAM by immersing it in 1 
$\mathrm{mM}$ 10-carboxy-1-decanethiol in ethanol for $30 \mathrm{~min}$ at room temperature. Subsequently, the electrode was rinsed with ethanol and double distilled water. The ITO electrode, a Ag/AgCl reference electrode (\#2080 A; Horiba, Japan), and a Pt auxiliary electrode were connected to a potentiostat (Autolab, PGSTAT12, Eco Chemie, The Netherlands). Cyclic voltammograms pertaining to desorption of the SAM were obtained with the three-electrode configuration in a $0.5 \mathrm{M} \mathrm{KOH}$ solution that was deoxygenated by bubbling nitrogen gas for 20 min prior to use. In this study, all potential values refer to those measured with respect to the Ag/AgCl electrode.

X-ray photo spectroscopy (XPS) spectra were obtained using a JPS-9010TR photoelectron spectrometer with a monochromatic AL Ka X-ray source (JEOL, Japan). Narrow scans were conducted to obtain the $S$ 2p peak of samples of the bare ITO electrode, ITO electrode modified with the SAM, and ITO electrode after desorption of the SAM by application of $-1.0 \mathrm{~V}$ for $5 \mathrm{~min}$.

Changes in surface topography following modification of ITO with the SAM were determined by atomic force microscopy (AFM) (5500 AFM, Agilent Technologies Inc., CA, USA) using the acoustic AC mode with a single crystal Si tip, a resonant frequency of $290.3 \mathrm{kHz}$ and a scan speed of $1 \mu \mathrm{m} / \mathrm{s}$. All images were obtained under atmospheric pressure at room temperature. Images were analyzed using the Pico Image Basic 
Software (Agilent Technologies Inc.).

\subsection{Preparation of cells}

Swiss 3T3 murine fibroblasts (RCB1642) were purchased from the Riken Cell Bank, Japan, and maintained in DMEM supplemented with 10\% FBS. The medium was changed every other day. Human umbilical vein endothelial cells (HUVECs, CC-2517A) were purchased from Cambrex Bio Science, USA, and maintained in EBM-2 supplemented with SingleQuots growth supplements. The medium was changed every other day. Each passage was conducted in a solution of $0.25 \%$ trypsin and $0.02 \%$ ethylenediamine tetraacetic acid (EDTA) after 3-4 d for fibroblasts and 2-3 d for HUVECs. Both cells were resuspended in fresh medium and diluted 1:3. HUVECs from passages 3 through 8 were used for the experiments.

\subsection{Modification of ITO electrode for cell culture}

To promote adhesion of the cells, the carboxyl terminals of the alkanethiol SAM were coupled to a cell adhesion peptide, GRGDS, through carbodiimide-mediated cross-linking as previously described (Fig. 1A) [14]. Growth of cells on the modified electrode was investigated using fibroblasts. Briefly, fibroblasts $\left(1.0 \times 10^{5}\right.$ cells $\left./ \mathrm{ml}\right)$ in 2 
$\mathrm{mL}$ of culture medium were seeded on a conventional culture dish, bare ITO electrode, and ITO electrode modified with the alkanethiol SAM and RGD. The cells were cultured for four days and the number was measured every day. The cell number was counted based on the number of nuclei using the crystal violet staining method [16].

\subsection{Electrochemical detachment of cells}

Fibroblasts $\left(1.0 \times 10^{5}\right.$ cells $\left./ \mathrm{ml}\right)$ in $2 \mathrm{~mL}$ of culture medium were seeded on the modified electrode, after which they were cultured for $12 \mathrm{~h}$ at $37^{\circ} \mathrm{C}$ in $5 \% \mathrm{CO}_{2}$ in a humidified incubator. The electrode was then washed with PBS three times and connected to a potentiostat (HA-151; Hokuto-Denko, Japan) in PBS. After applying a potential of-1.0 $\mathrm{V}$, the electrode was washed gently with PBS using a pipette, and the cells that remained on the substrate were counted by taking phase-contrast micrographs. For comparison, the same experiments were conducted under conditions in which the cells were attached directly to a bare ITO electrode or no potential was applied.

\subsection{Change in cell morphology during electrochemical detachment}

Cellular morphology before and after the detachment was investigated using a confocal microscope. Fibroblasts were cultured for $24 \mathrm{~h}$ on the modified ITO electrode. Cells 
before and after the potential application were fixed with 3.7\% formaldehyde in PBS for $10 \mathrm{~min}$, after which they were permeabilized with $0.2 \%$ Triton X-100 for 5 min. The cells were then incubated with a mixed solution of rhodamine phalloidin and DAPI for 20 min, after which they were rinsed with PBS for 1 min. Images were taken using a confocal microscope (FV1000-D, Olympus, Japan) with a laser diode as the excitation light source for rhodamine-phalloidin and DAPI.

\subsection{Micropatterning of ITO electrode and spatially selective detachment of cells}

Micropatterns of ITO electrodes were fabricated using photolithography [17]. Briefly, an approximately 100-nm-thick gold layer was initially sputter-coated on an ITO layer by a RF magnetron sputtering (MSP 30T; Vacuum Device, Japan). Next, a positive photoresist was spin-coated onto the gold layer and exposed to UV light through a photomask using a mask aligner (MA-10; MIKASA, Japan). The photoresist patterns were then developed in MF-319 solution for $5 \mathrm{~min}$, after which the samples were washed with DI water and dried. The gold layer was then etched by immersion in an aqueous solution containing $0.1 \mathrm{~g} / \mathrm{ml}$ potassium iodide and $25 \mathrm{mg} / \mathrm{ml}$ iodine for $5 \mathrm{~min}$. Subsequently, the ITO layer was etched by immersion in an aqueous solution containing 28\% (v/v) nitric acid and 31\% (v/v) hydrochloric acid for 8 min. The remaining 
photoresist and gold layer were completely removed using the acetone and etching solution. The micropatterned ITO electrode was then modified with the alkanethiol SAM and RGD peptide using the same procedures described earlier. Fibroblasts were seeded onto the electrode and cultured for $24 \mathrm{~h}$. The cells on the electrode micropattern were then sequentially detached by applying $-1.0 \mathrm{~V}$ for $5 \mathrm{~min}$.

\section{Results and discussion}

\subsection{Electrical desorption of SAMs}

Alkanethiol SAMs have been extensively examined on gold to investigate the effects of surface nature, including surface wettability and charges, on protein adsorption and cell adhesion $[18,19]$. Despite a large body of literature regarding alkanethiol SAMs on gold, studies regarding ITO are relatively rare [20, 21]. In this decade, the use of ITO has promoted the revolution in electronics through their use in items such as touch panels and solar batteries. The preferable features of transparent ITO may also be beneficial for cell-based systems.

The ITO surface structure largely depends on the fabrication condition, which influences the formation of a well-packed and ordered SAM and reproducibility of experiments [20]. The surface topography of ITO was examined using AFM prior to use. 
The ITO surfaces presented crystalline grains (Fig. 2A). ITO with a maximal height difference less than $25 \mathrm{~nm}$ was used for this study. It should be noted that on the basis of AFM analysis the surface topography was not significantly altered by the treatment processes for the modification with 10-carboxy-1-decanethiol in ethanol (Fig. 2A) or the electrical desorption of the SAM (data not shown). The potential required for desorption of the SAM from ITO was estimated using cyclic voltammetry. As shown in Fig. 2B, a peak potential appeared at approximately $-0.9 \mathrm{~V}$. Although the interaction on an atomic level between ITO and thiol (unlike gold and thiol) has not yet been clarified, this value is very close to that observed on a gold surface in our previous study $[14,15]$. XPS measurements were conducted to investigate changes in chemical bonding in the formation and desorption of the SAM on ITO [21]. Fig. 2C shows the high-resolution XPS spectra for bare ITO, ITO modified with the SAM, and ITO electrode after desorption of the SAM by application of $-1.0 \mathrm{~V}$ for 5 min. A clear peak corresponding to the S 2p orbital at $162 \mathrm{eV}$ was observed for the SAM-modified ITO, whereas no peak was observed for the bare ITO or the ITO modified with the SAM and treated with an electrical potential. These electrochemical and XPS results indicate that the alkanethiol spontaneously adsorbed onto the ITO electrode and was then reductively desorbed by application of the potential. Based on these results, we decided to use $-1.0 \mathrm{~V}$ for 
desorption of the SAM and detachment of cells in the following experiments.

\subsection{Change in cell morphology during electrochemical detachment}

The ITO electrode modified with the SAM and RGD peptide was used to investigate cell proliferation and the electrochemical detachment of cells. Fibroblasts were readily attached and spread out on the modified ITO electrode (Fig. 3A). The number of cells was then counted every day for four days. The proliferation of fibroblasts on the electrode (doubling time, $20 \pm 3.5 \mathrm{~h}$ ) was slightly slower than that on a typical culture dish $(16 \pm 1.5 \mathrm{~h})$ and that reported in the literature $(16.6 \mathrm{~h})$, but these differences were not significant [22]. At $24 \mathrm{~h}$ of culture, fibroblasts adhering to the electrode were detached by applying a potential of $-1.0 \mathrm{~V}$. Figure $3 \mathrm{~B}$ shows the change in cell morphology at 40-s intervals during the detachment. The cells were gradually detached from their edges and appeared bright after application of the potential, after which they were identified for detachment by gentle pipetting (Fig. 3C). Changes in the cell shape were also observed and characterized by confocal microscopy after staining of the F-actin and nuclei (Fig. 4). The top and side views show that the extended cells had a bulge at the nucleus and stress fibers (Figs. 4A and B). Image analysis of these cells revealed that the approximate height and width before application of the potential were 
$7.9 \mu \mathrm{m}$ and $74 \mu \mathrm{m}$, respectively (Figs. 4E and F). These cells were rounded after applying the potential (Figs. 4C and D). In this process, the width decreased to approximately $13 \mu \mathrm{m}$, whereas the height only increased to about $12 \mu \mathrm{m}$ (Fig. 4E and F). It should be noted that Figs. 4A and 4C represent full stacks of confocal images and Figs. 4B and 4D represent stacks of images from the bottom to the middle of the cell. The dynamic cell attachment/detachment to and from extracellular microenvironments in vivo regulates cell growth, differentiation, and apoptosis [23]. The relationships between the formation of focal adhesion complexes at the interface and a wide range of signal transduction and gene expression have typically been examined using the adhesion process of suspended cells [24]. This approach could potentially enable focal adhesion disassembly and subsequent remodeling of the cytoskeleton using the cell detachment process.

\subsection{Quantitative analysis of the detachment of cells}

To quantitatively analyze the detachment of cells, the number of fibroblasts that remained on the surface following application of the potential was counted at 60-s intervals (Fig. 4G). Cells were attached to the bare ITO well probably due to adhesion proteins in FBS and there was no significant difference in the number of cells initially 
attached onto the ITO electrode with or without modification with the SAM and RGD

(1.5 to $2 \times 10^{4}$ cells $/ \mathrm{cm}^{2}$ ). When the SAM and an electrical potential of $-1.0 \mathrm{~V}$ were used, more than $90 \%$ of the cells were detached from the surface within 5 min. To the best of our knowledge, this is the most rapid cell detachment approach that has been reported to date. In the control experiments (no SAM or no potential application), the behavior of the cells was clearly different as shown in Fig. 4G. These results suggest that electrical desorption of the SAM is primarily responsible for the detachment of cells. Note that approximately $30 \%$ of the cells were detached in the control experiment (conditions of no SAM and $-1.0 \mathrm{~V}$ ). This was likely because some cells attached onto the bare ITO via proteins and were detached together with proteins in response to application of the potential.

\subsection{Spatially controlled detachment of cells}

To selectively detach cells on demand, a micropattern of ITO electrodes was fabricated via wet etching and the electrodes were then modified with the SAM and RGD peptide. The spacing between the electrodes was $50 \mu \mathrm{m}$. As shown in Fig. 5, cells adhering to the ITO microelectrodes were clearly observed upon phase-contrast microscopy. Cells adhering onto the microelectrodes were electrically detached one by one. A cell on the 
electrode labeled 1 was detached by application of the potential for 5 min as shown in Fig. 5B. It should be noted that there was no influence on the cells on the neighboring electrodes during this detachment. Similarly, cells on the other electrodes were detached sequentially in numerical order as shown in Figs. 5B-5D.

The selective cell detachment could potentially be useful in fundamental cell biology as well as cell processing for regenerative medicine. In the field of regenerative medicine, considerable efforts have been focused on inducing differentiation of iPS cells or ES cells to a specific cell type [25]. However, the multipotency of stem cells makes this process difficult. Because our technology has enabled access to cells being observed under a microscope, it may be possible to alleviate this issue by eliminating undesired cell populations from culture based on estimation of cell fate from their shapes on a culture surface. In the present study, we only fabricated four microelectrodes as proof of concept. However, in the semiconductor industry, the precision of microfabrication technologies has already reached the nano meter scale. Our next goal is to fabricate ITO microelectrodes with dimensions smaller than those of a cell.

\section{Conclusion}

We revealed that an alkanethiol molecule spontaneously adsorbs onto ITO, and can then 
be reductively desorbed by the application of potential. Using this dynamic reaction, cells were detached in a reliable and rapid manner, with more than $90 \%$ of adherent cells being electrically detached by application of potential for 5 min. Furthermore, owing to the transparency of ITO, cells on the microarray of ITO electrodes could be clearly observed using conventional phase-contrast microscopy and were selectively detached on-demand. Although this is a rapid cell detachment approach, considering that desorption of the SAM was completed within one minute during cyclic voltammetry analysis, there may be room for acceleration of the detachment of cells. This study did not focus on the crystalline architecture of ITO or orientation of SAM, which may be largely responsible for the electrical cell detachment. It still remains to be seen whether atomically flat ITO epitaxial film can hasten the processes described herein [26]. This electrical cell detachment could be a fundamental tool in diverse research fields, ranging from fundamental cell biology to tissue engineering applications.

\section{Acknowledgments}

This study was supported by the Ministry of Education, Culture, Sports, Science and 
Technology (Grant-in-Aid for Young Scientists (A), 20686056), the Ministry of Health, Labor and Welfare (H20-Saisei-wakate-010), and NEDO (Industrial Technology Research Grant Program, 06A06014a).

\section{References}

1. Langer R, Tirrell DA. Designing materials for biology and medicine. Nature 2004;428(6982):487-492.

2. Lahann J, Mitragotri S, Tran TN, Kaido H, Sundaram J, Choi IS, et al. A reversibly switching surface. Science 2003;299(5605):371-374.

3. Matsuda N, Shimizu T, Yamato M, Okano T. Tissue engineering based on cell sheet technology. Adv Mater 2007;19(20):3089-3099.

4. Fukuda J, Khademhosseini A, Yeh J, Eng G, Cheng JJ, Farokhzad OC, et al. Micropatterned cell co-cultures using layer-by-layer deposition of extracellular matrix components. Biomaterials 2006;27(8):1479-1486.

5. Cole MA, Voelcker NH, Thissen H, Griesser HJ. Stimuli-responsive interfaces and systems for the control of protein-surface and cell-surface interactions. Biomaterials 2009;30(9):1827-1850.

6. Nandivada H, Ross AM, Lahann J. Stimuli-responsive monolayers for 
biotechnology. Progress Polym Sci 2010;35(1-2):141-154.

7. Elloumi-Hannachi I, Yamato M, Okano T. Cell sheet engineering: a unique nanotechnology for scaffold-free tissue reconstruction with clinical applications in regenerative medicine. J Intern Med 2010;267(1):54-70.

8. Nishida K, Yamato M, Hayashida Y, Watanabe K, Yamamoto K, Adachi E, et al. Corneal reconstruction with tissue-engineered cell sheets composed of autologous oral mucosal epithelium. N Engl J Med 2004;351(12):1187-1196.

9. Kamihira M, Akiyama H, Ito A, Kawabe Y. Genetically engineered angiogenic cell sheets using magnetic force-based gene delivery and tissue fabrication techniques. Biomaterials 2010;31(6):1251-1259.

10. Yeo WS, Mrksich M. Electroactive self-assembled monolayers that permit orthogonal control over the adhesion of cells to patterned substrates. Langmuir 2006;22(25):10816-10820.

11. Guillaume-Gentil O, Akiyama Y, Schuler M, Tang C, Textor M, Yamato M, et al. Polyelectrolyte coatings with a potential for electronic control and cell sheet engineering. Adv Mater 2008;20(3):560-565.

12. Guillaume-Gentil O, Gabi M, Zenobi-Wong M, Voros J. Electrochemically switchable platform for the micro-patterning and release of heterotypic cell sheets. 
Biomed Microdevices 2011;13(1):221-230.

13. Kim M, Lee JY, Shah SS, Tae G, Revzin A. On-cue detachment of hydrogels and cells from optically transparent electrodes. Chemical Communications 2009(39):5865-5867.

14. Inaba R, Khademhosseini A, Suzuki H, Fukuda J. Electrochemical desorption of self-assembled monolayers for engineering cellular tissues. Biomaterials 2009;30(21):3573-3579.

15. Seto Y, Inaba R, Okuyama T, Sassa F, Suzuki H, Fukuda J. Engineering of capillary-like structures in tissue constructs by electrochemical detachment of cells. Biomaterials 2010;31(8):2209-2215.

16. Gillies RJ, Didier N, Denton M. Determination of Cell Number in Monolayer-Cultures. Anal Biochem 1986;159(1):109-113.

17. Suzuki M, Yasukawa T, Shiku H, Matsue T. Separation of live and dead microorganisms in a micro-fluidic device by dielectrophoresis. Bunseki Kagaku 2005;54(12):1189-1195.

18. Tredgold RH. An Introduction to Ultrathin Organic Films - from Langmuir-Blodgett to Self-Assembly - Ulman,A. Nature 1991 Nov 14;354(6349):120-120. 
19. Sigal GB, Mrksich M, Whitesides GM. Effect of surface wettability on the adsorption of proteins and detergents. J Am Chem Soc 1998;120(14):3464-3473.

20. Cerruti M, Rhodes C, Losego M, Efremenko A, Maria JP, Fischer D, et al. Influence of indium-tin oxide surface structure on the ordering and coverage of carboxylic acid and thiol monolayers. Journal of Physics D-Applied Physics 2007;40(14):4212-4221.

21. Yan C, Zharnikov M, Golzhauser A, Grunze M. Preparation and characterization of self-assembled monolayers on indium tin oxide. Langmuir 2000;16(15):6208-6215.

22. Rossow PW, Riddle VG, Pardee AB. Synthesis of labile, serum-dependent protein in early G1 controls animal cell growth. Proc Natl Acad Sci USA 1979;76(9):4446-4450.

23. Huang S, Ingber DE. Shape-dependent control of cell growth, differentiation, and apoptosis: switching between attractors in cell regulatory networks. Exp Cell Res 2000;261(1):91-103.

24. Dobereiner HG, Dubin-Thaler B, Giannone G, Xenias HS, Sheetz MP. Dynamic phase transitions in cell spreading. Phys Rev Lett 2004;93(10):4.

25. Snykers S, De Kock J, Rogiers V, Vanhaecke T. In vitro differentiation of 
embryonic and adult stem cells into hepatocytes: state of the art. Stem Cells 2009;27(3):577-605.

26. Ohta H, Orita M, Hirano M, Tanji H, Kawazoe H, Hosono H. Highly electrically conductive indium-tin-oxide thin films epitaxially grown on yttria-stabilized zirconia (100) by pulsed-laser deposition. Appl Phys Lett 2000;76(19):2740-2742. 


\section{Figure legends}

Fig. 1 Detachment of cells in a spatially and temporally controlled manner. (A) Cross-sectional view of micropatterned ITO electrodes. The electrodes were modified with an alkanethiol SAM and RGD peptide. A cell on a specific electrode was detached by applying a negative potential. (B) Top view indicating microelectrodes, electrode leads and pads for the connections to a potentiostat. The schematic is not equivalent to the real dimensions and the cells and microelectrodes were drawn enlarged for clarity of illustration.

Fig. 2 Characterizations of ITO modified with the SAM. (A) AFM images of bare ITO and ITO after adsorption of the oligopeptide. The scanning areas are $1 \times 1 \mu \mathrm{m}$. (B) Cyclic voltammogram observed during reductive desorption of the SAM. Cyclic voltammograms were recorded at a scanning rate of $20 \mathrm{mV} / \mathrm{s}$ with respect to a $\mathrm{Ag} / \mathrm{AgCl}$ reference electrode. The working electrode area is $0.4 \mathrm{~cm}^{2}$. (C) XPS spectra observed for samples of bare ITO, ITO modified with the alkanethiol SAM, and ITO after the application of $-1.0 \mathrm{~V}$ for $5 \mathrm{~min}$.

Fig. 3 Detachment of fibroblasts from the ITO electrode. (A) Cells attached to the ITO 
electrode modified with the SAM and RGD peptide and cultured for $24 \mathrm{~h}$. (B) Changes in cell morphology during the application of $-1.0 \mathrm{~V}$ were monitored at 40 -s intervals. (C) After application of potential for 5 min the cells were identified for detachment by gentle pipetting.

Fig. 4 Changes in cell shape of fibroblasts and quantitative analysis of cell detachment. (A-D) Representative images of a fibroblast before (A and B) and after (C and D) application of potential. Rhodamine-phalloidin was used to stain actin fibers red and DAPI was used to stain nuclei blue. Side views (A and C) indicate images constructed by stacking optically sectioned images of an entire cell from edge to edge, and top views ( $\mathrm{B}$ and $\mathrm{D}$ ) indicate images constructed by stacking images from the bottom to the middle of the cell. (E, F) Image analysis was conducted to obtain quantitative data regarding the cell height (E) and width (F) before and after the potential application. The values indicate the mean of four independent experiments. Error bars indicate the SD. (G) Changes in the number of fibroblasts remaining on the ITO electrode. More than $90 \%$ of the cells were detached from the surface after application of the potential for 5 min along with desorption of the SAM (SAM,-1.0 V). Significantly lower numbers of cells were detached from the surface when the cells were attached directly 
onto a bare ITO electrode (no SAM, $0 \mathrm{~V}$ ) or when no potential was applied (SAM, $0 \mathrm{~V}$ ).

The values indicate the mean of nine independent experiments. Error bars indicate the SD.

Fig. 5 Selective detachment of cells using a micropatterned ITO electrode. HUVECs attached onto the microelectrodes were sequentially detached by applying the potential to the electrodes in the numerical order of labeled digits. The spacing between the electrodes was $50 \mu \mathrm{m}$. (A) The circle labeled 1 indicates the initially activated electrode. (B) Cells on the electrode labeled 1 were detached. The circle labeled 2 indicates the subsequently activated electrode. (C) The cells on the electrode labeled 2 were detached. The circle labeled 3 indicates the activated electrode. (D) The cell on the electrode labeled 3 was detached. 
Fig. 1 J. Fukuda, et al.

A

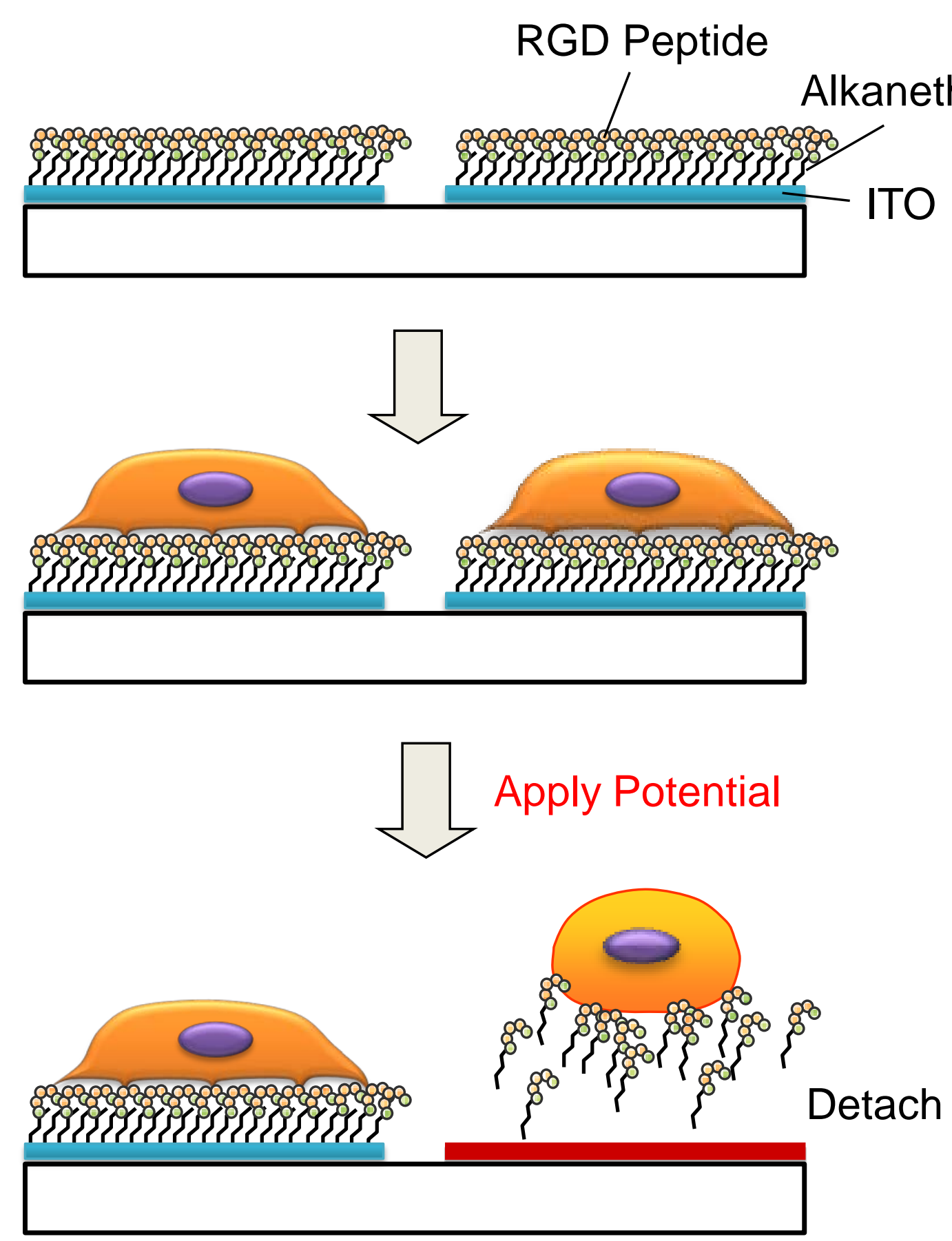

B

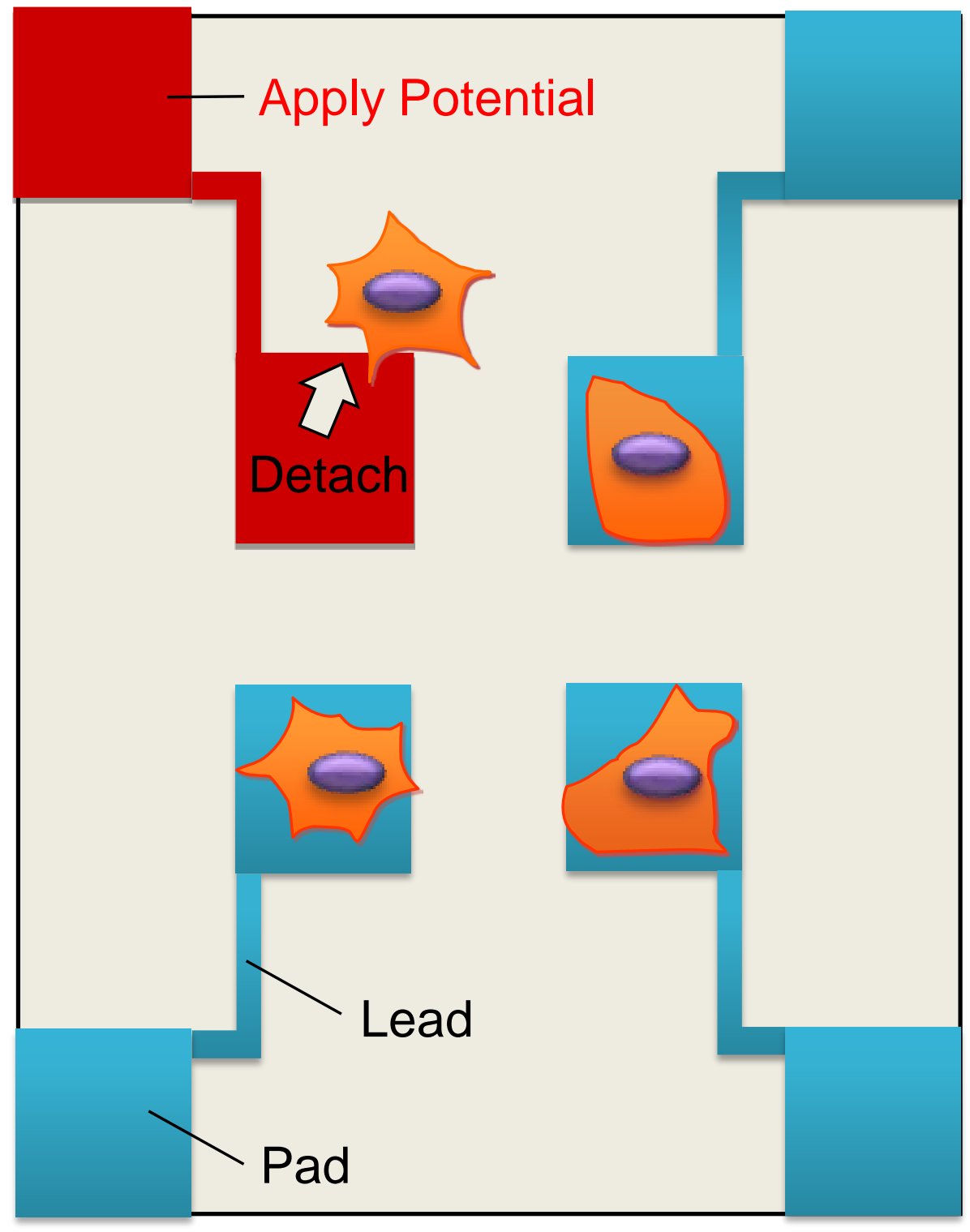


Fig. 2 J. Fukuda, et al.

A
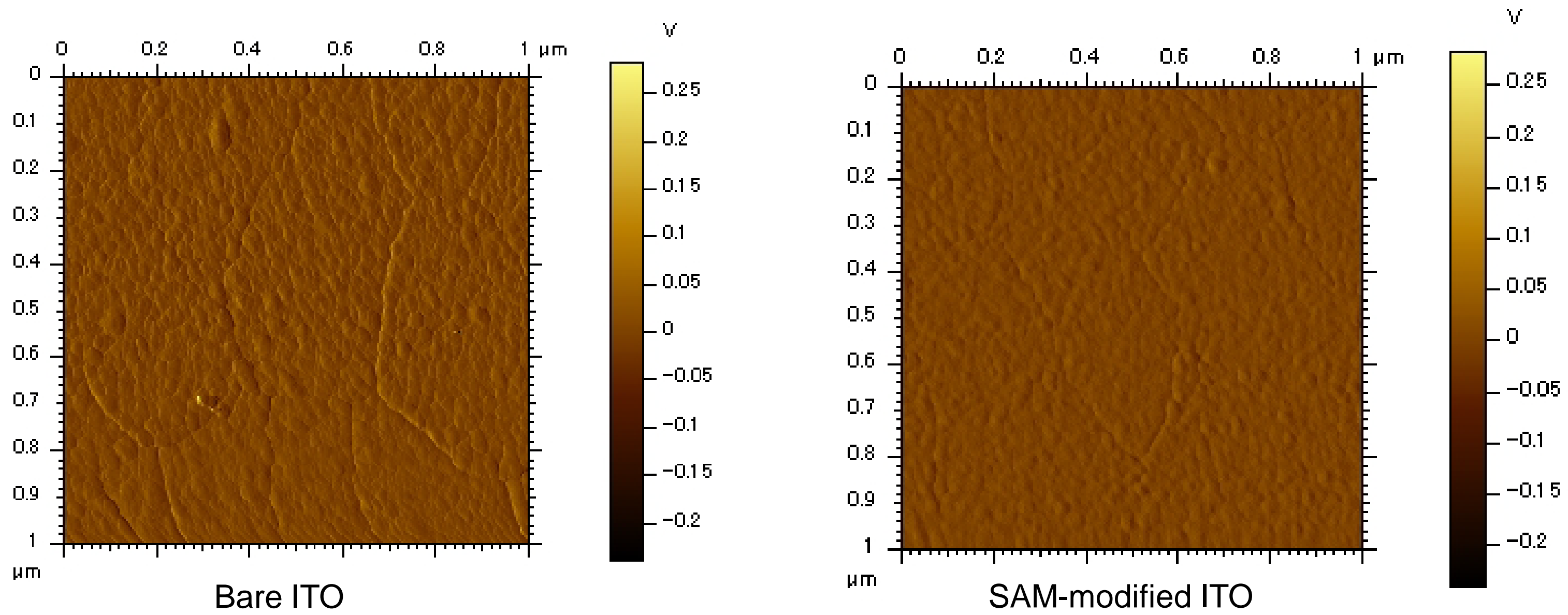

B

C
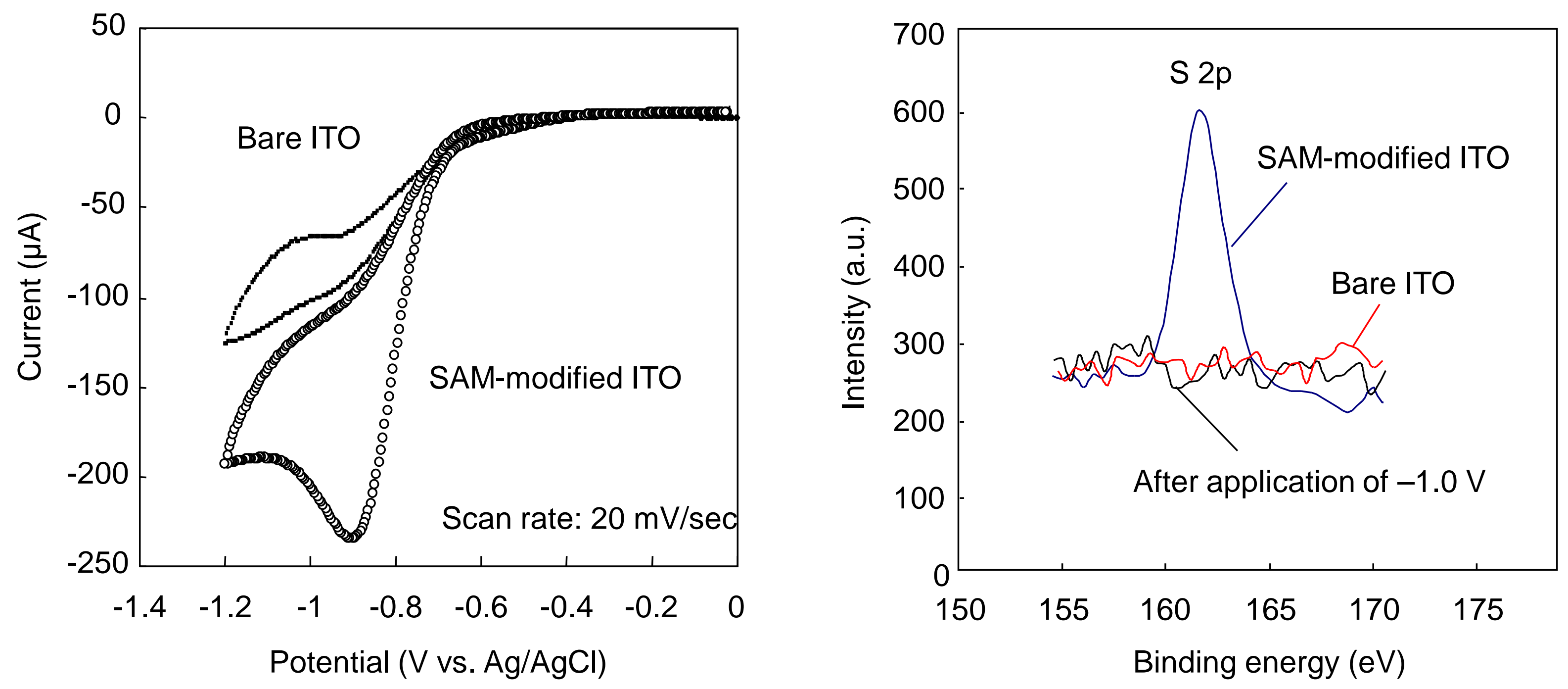
Fig. 3 J. Fukuda, et al.
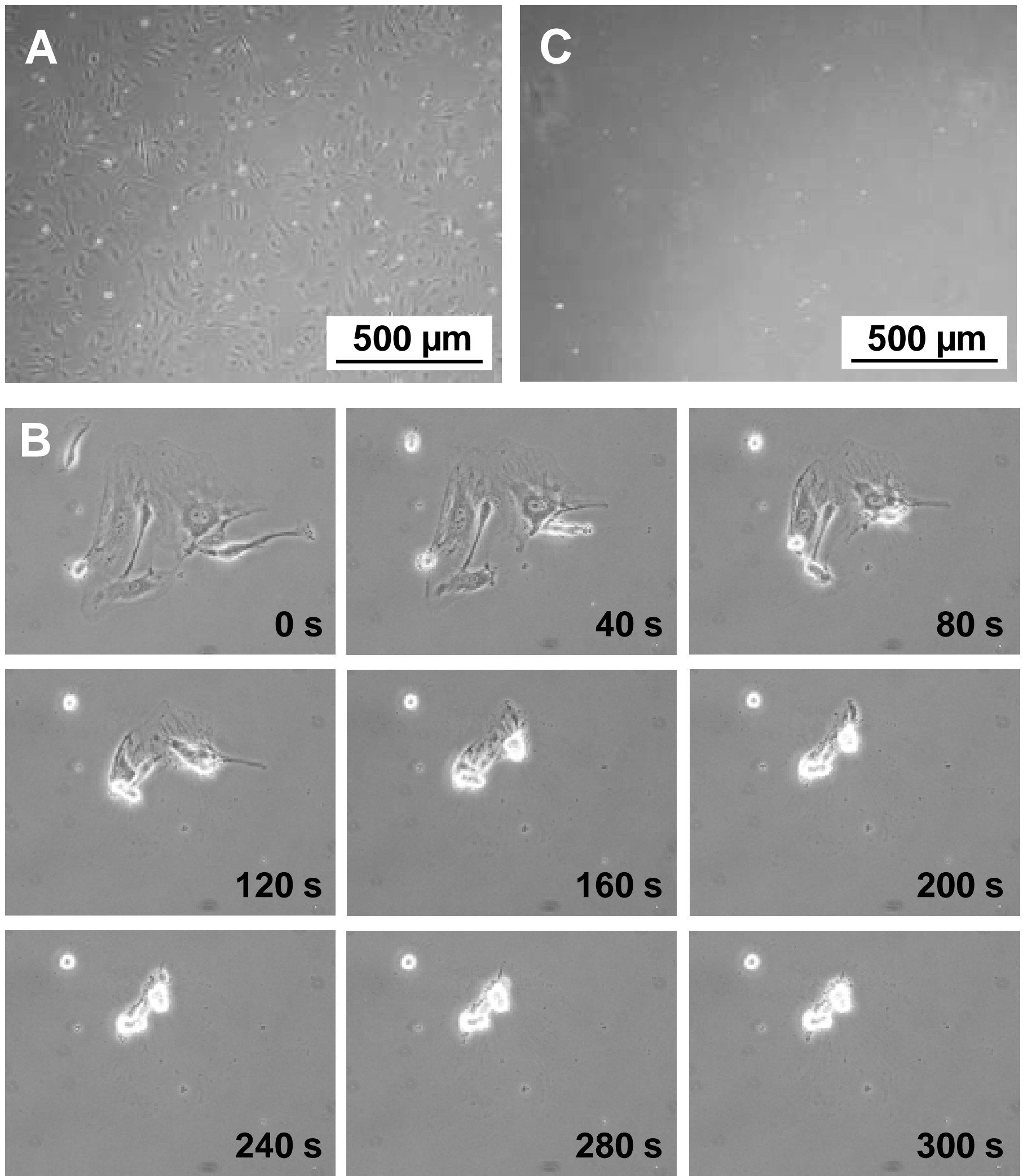

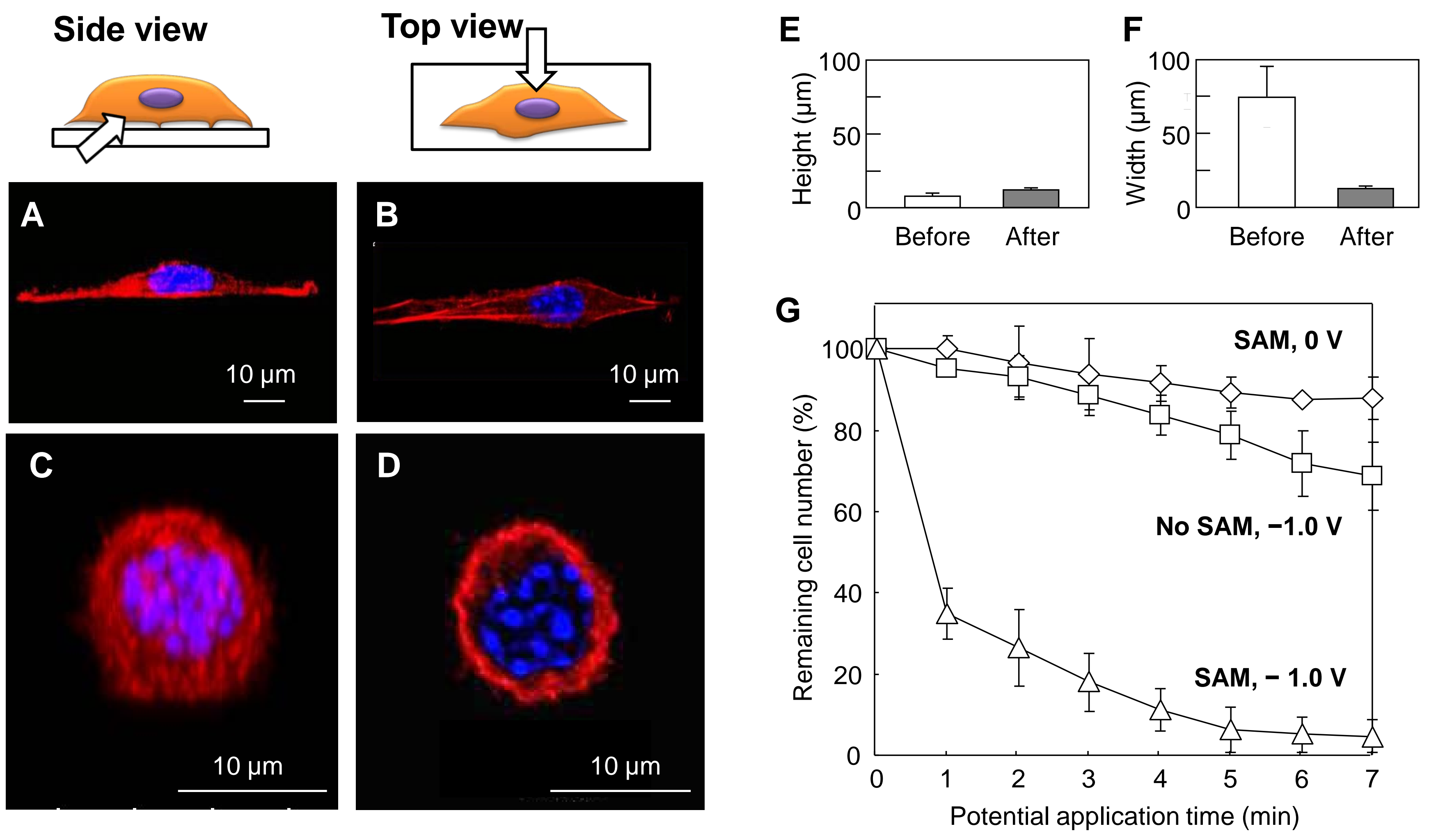
Fig. 5 J. Fukuda, et al.

A
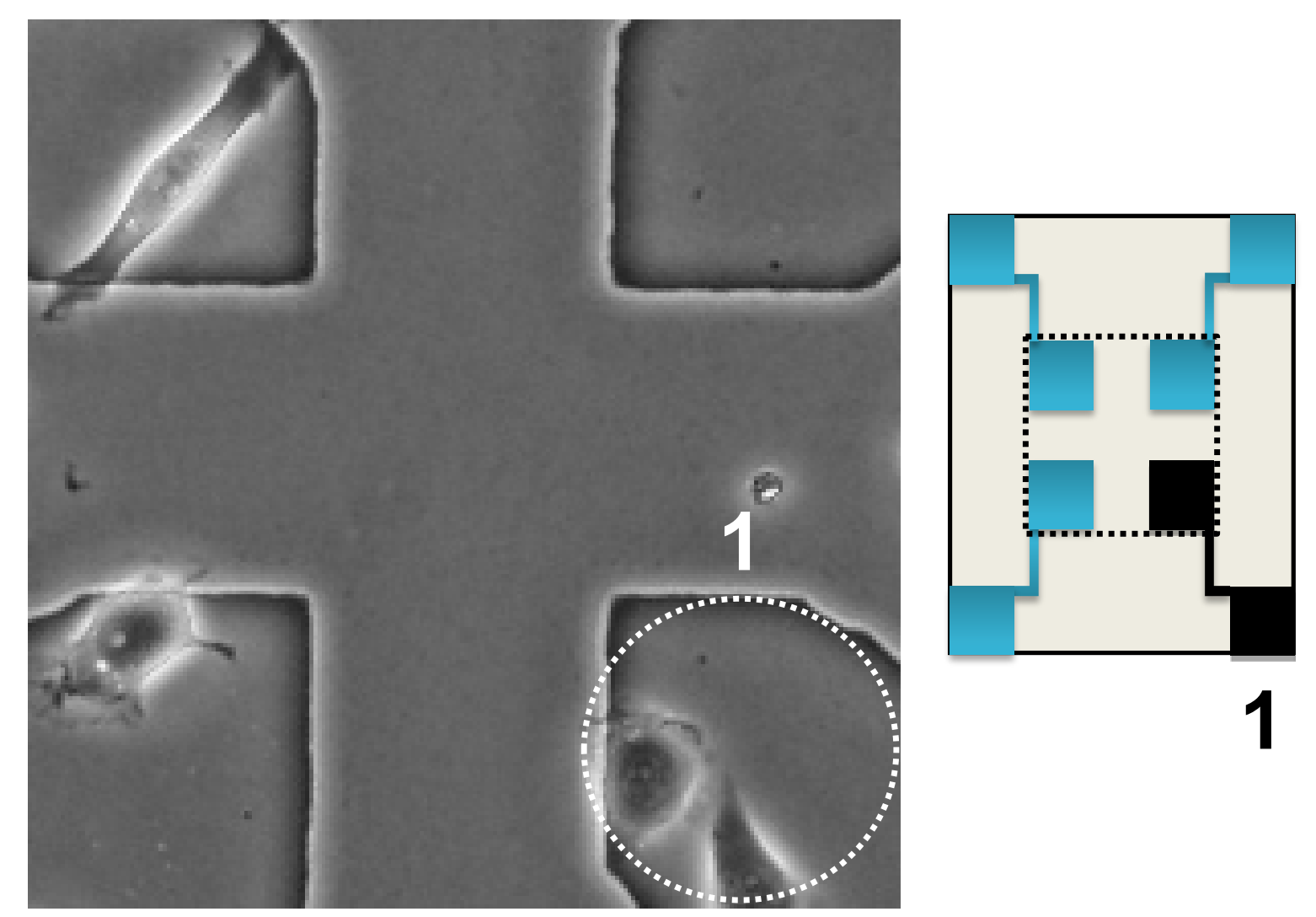

C
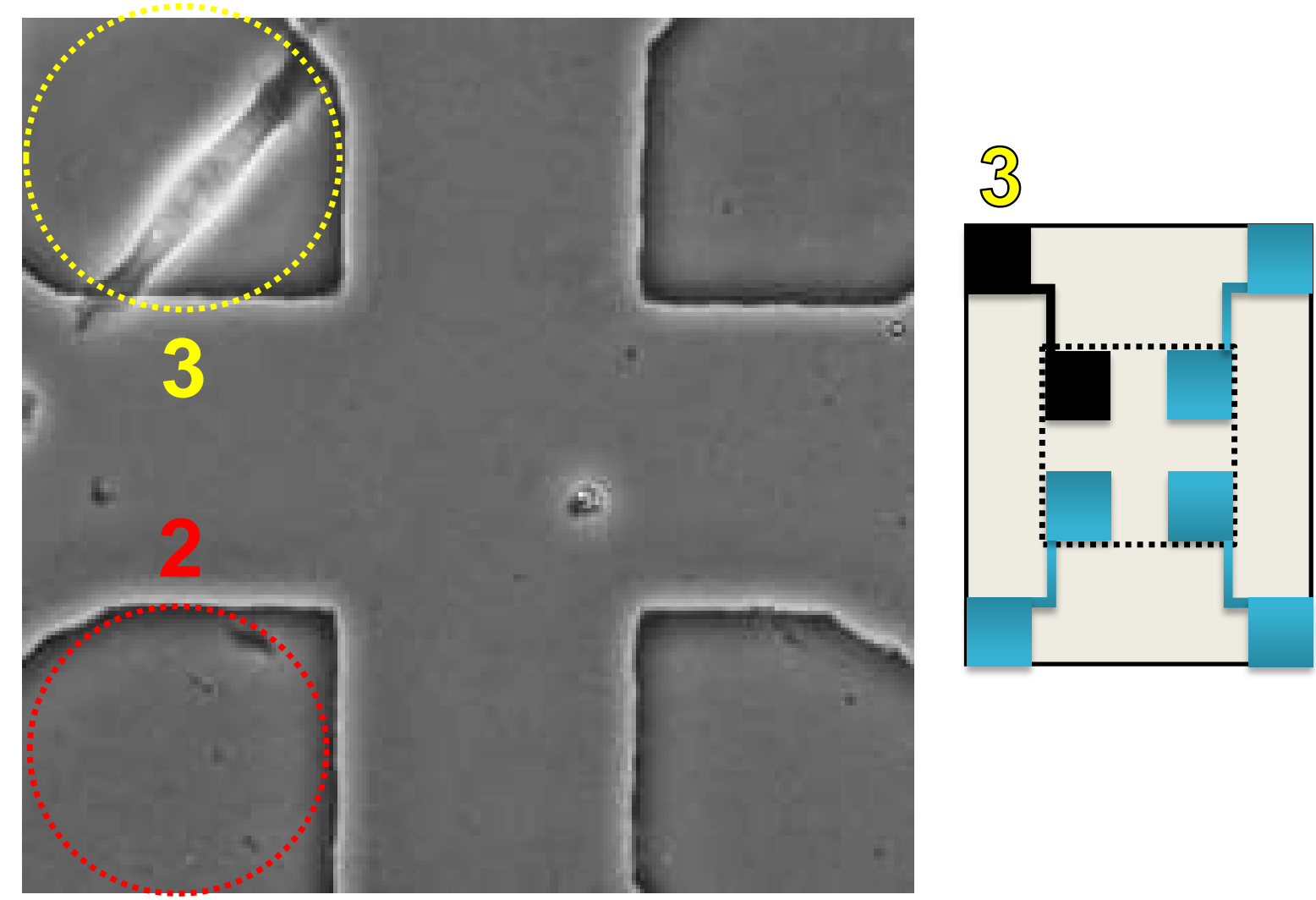

B
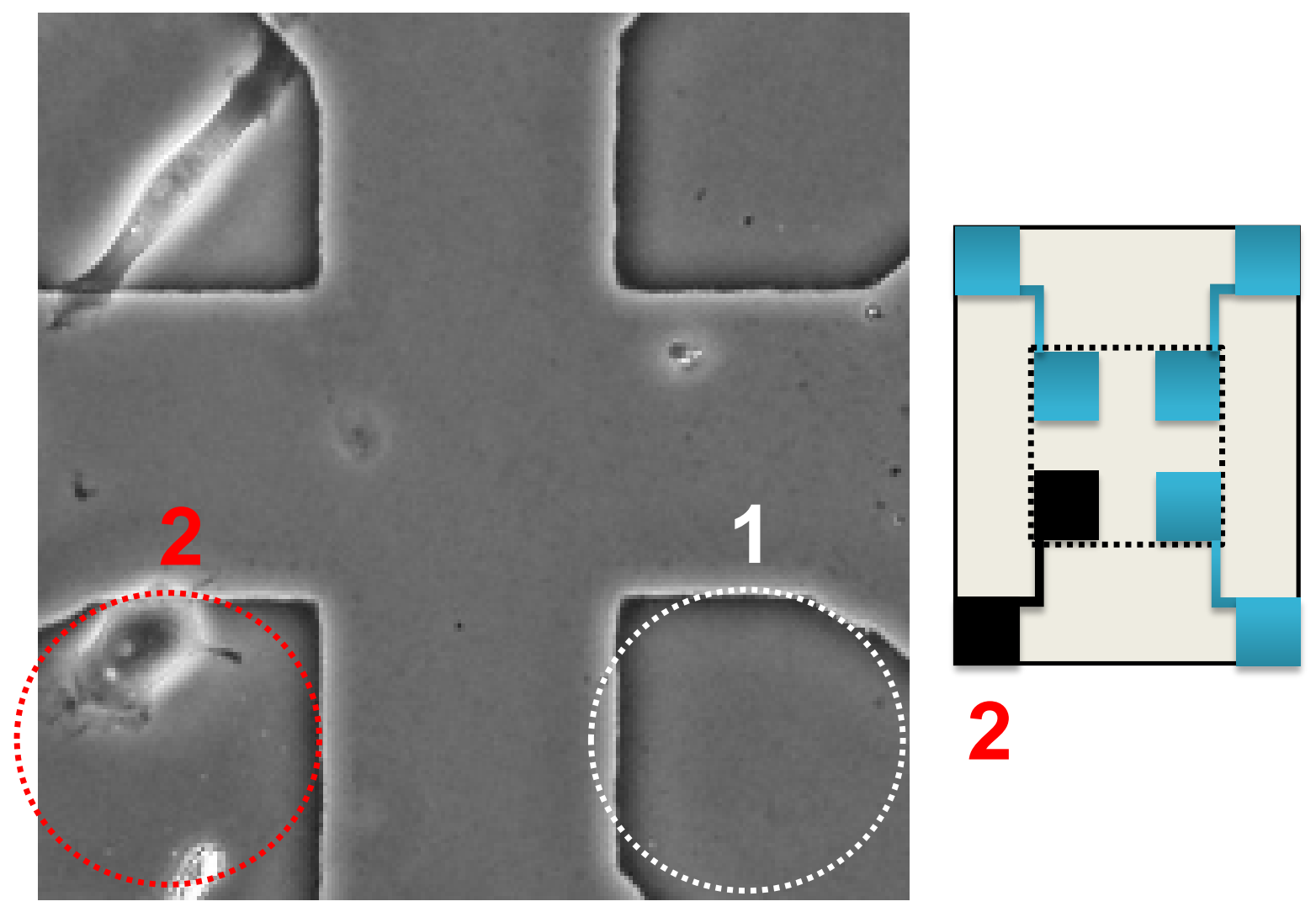

D

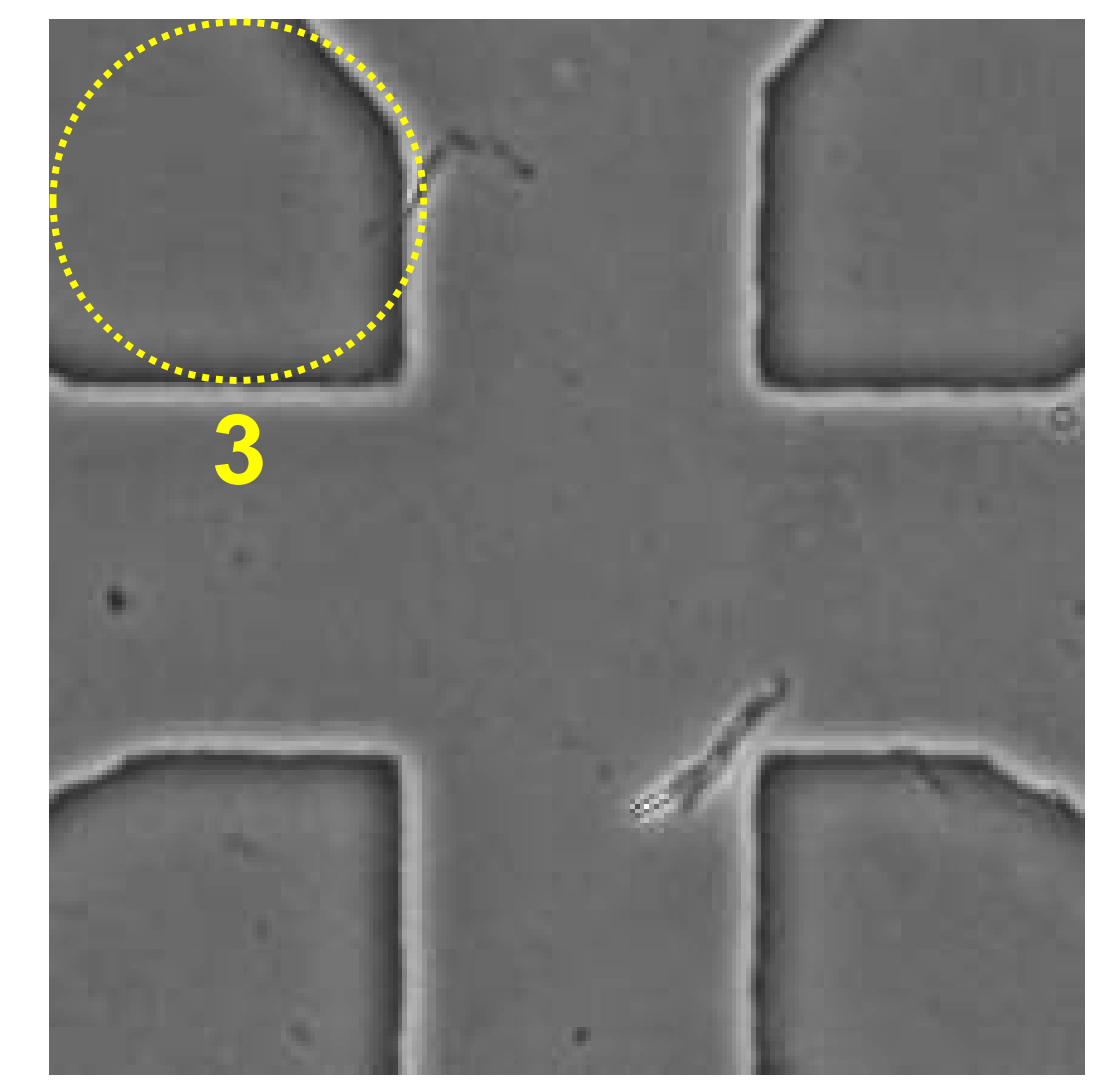


Supplemental Fig. 1 J. Fukuda, et al.

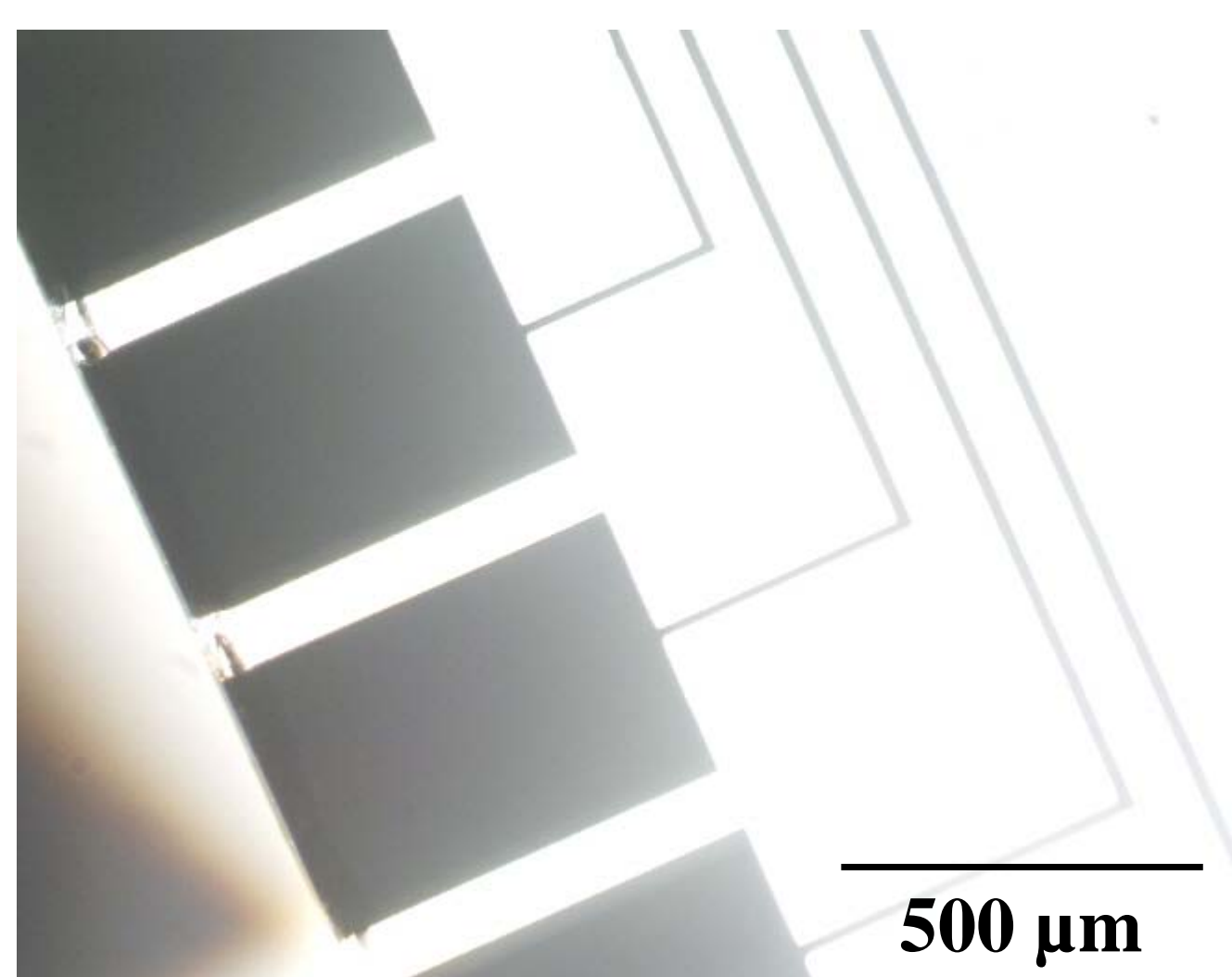

A phase-contrast micrograph showing halation caused through a glass substrate with a micropattern of gold electrodes. 